\title{
Interpreting the Regulatory Interplay in $E$. coli Metabolic Pathways
}

\author{
Anália Lourenço*, Sónia Carneiro*, José P. Pinto, Miguel Rocha,
} Eugénio C. Ferreira, and Isabel Rocha

\begin{abstract}
Many regulatory processes in the cell are based on the control of gene expression through the interaction of transcription factors. However, enzymatic regulation often overlays transcriptional regulation and even, in some metabolic pathways, enzymatic regulation prevails. The present study addresses the regulatory network of Escherichia coli and offers a global view of the regulation of its metabolic pathways. It identifies the regulatory mechanisms responsible for key metabolic activities and details the structures behind such mechanisms. This knowledge is considered of relevance to further studies on the bacteria's system and its industrial application, namely for understanding the signal cascades comprised in the responses to various environmental stresses.
\end{abstract}

\section{Introduction}

The analysis of biological networks aims at the understanding of metabolic capabilities of cells to adapt to, and to maintain growth under different external and internal conditions $[1,2]$. A particularly challenging task is the inference of the regulatory interactions commanding the activity of metabolic pathways. Different mechanisms are recruited for regulation, either long-term regulation by changing the expression level of genes or short-term regulation by changing the activity of enzymes.

Gene expression is mostly controlled by transcription factors (TFs) that are proteins able to bind to gene promoter regions, inducing or repressing the initiation of

\footnotetext{
Anália Lourenço $\cdot$ Sónia Carneiro $\cdot$ Eugénio C. Ferreira $\cdot$ Isabel Rocha

IBB - Institute for Biotechnology and Bioengineering, Centre of Biological Engineering e-mail: \{analia, soniacarneiro, ecferreira, irocha\} @deb.uminho.pt

José P. Pinto $\cdot$ Miguel Rocha

Department of Informatics / CCTC, University of Minho, Campus de Gualtar, 4710-057

Braga - Portugal

e-mail: \{josepedr, mrocha\} @di.uminho.pt

* These authors equally contributed to this work.
} 
gene transcription. In turn, the activity of enzymes, proteins that catalyze biochemical reactions, can be controlled by some effector molecules binding at the active or allosteric sites or by alteration of some environmental condition (e.g., pH or ionic strength).

In this work, we focus on the study of how transcriptional regulation couples with the regulation of the activity of metabolic pathways via enzymatic regulation, and how similar regulatory mechanisms are used across different metabolic pathways, i.e. the identification of regulatory circuits dominating certain pathway activities. Our goal is to obtain a global view of the regulatory interplay affecting the metabolism of the bacterium Escherichia coli (E. coli) K12, considering its common use as an industrial organism [3].

\section{Methodology}

The construction of a network integrating genome-scale transcriptional and enzymatic regulation requires information on gene encoding, gene regulation, genereaction associations, and enzymatic regulation. For this purpose, we can take previously validated metabolic and regulatory networks and/or retrieve information from publicly available repositories, and we need to perform the necessary data integration.

Here, we considered the gene-reaction associations from the genome-scale metabolic network of E. coli K12 (iAF1260) [4], and derived information on gene transcription regulation from the EcoCyc database [5]. Namely, TF-encoding genes that regulate the expression of metabolic genes (i.e. genes associated with a reaction from the $i \mathrm{AF} 1260$ model) and/or regulate other TF-encoding genes were included. Information on the control of enzyme activity was also obtained from EcoCyc and further associated with the respective enzyme-coding genes.

\subsection{Network Analysis}

Our network can be viewed as a graph with two types of nodes, genes and metabolites, and two types of edges: one that connects metabolites to genes that encode for enzymes they regulate; and another that connects pairs of genes that are linked by transcriptional regulation.

Using the representation provided by the Java Universal Network/Graph Framework (JUNG) (http://jung.sourceforge.net/), we analysed the properties of the graph. Specifically, the frequency of occurrence of different types of edges associated with metabolic genes determined the overlapping of transcriptional and enzymatic regulation, whereas regulatory patterns commonly described in literature $[6,7]$ provided deeper understanding on the interplays taking place on particular pathways (see details in Box 1).

To assess the importance of different regulatory motifs in particular metabolic pathways, we calculated the prevalence of motif types per pathway and the average number of genes in the pathway participating in such motifs. 


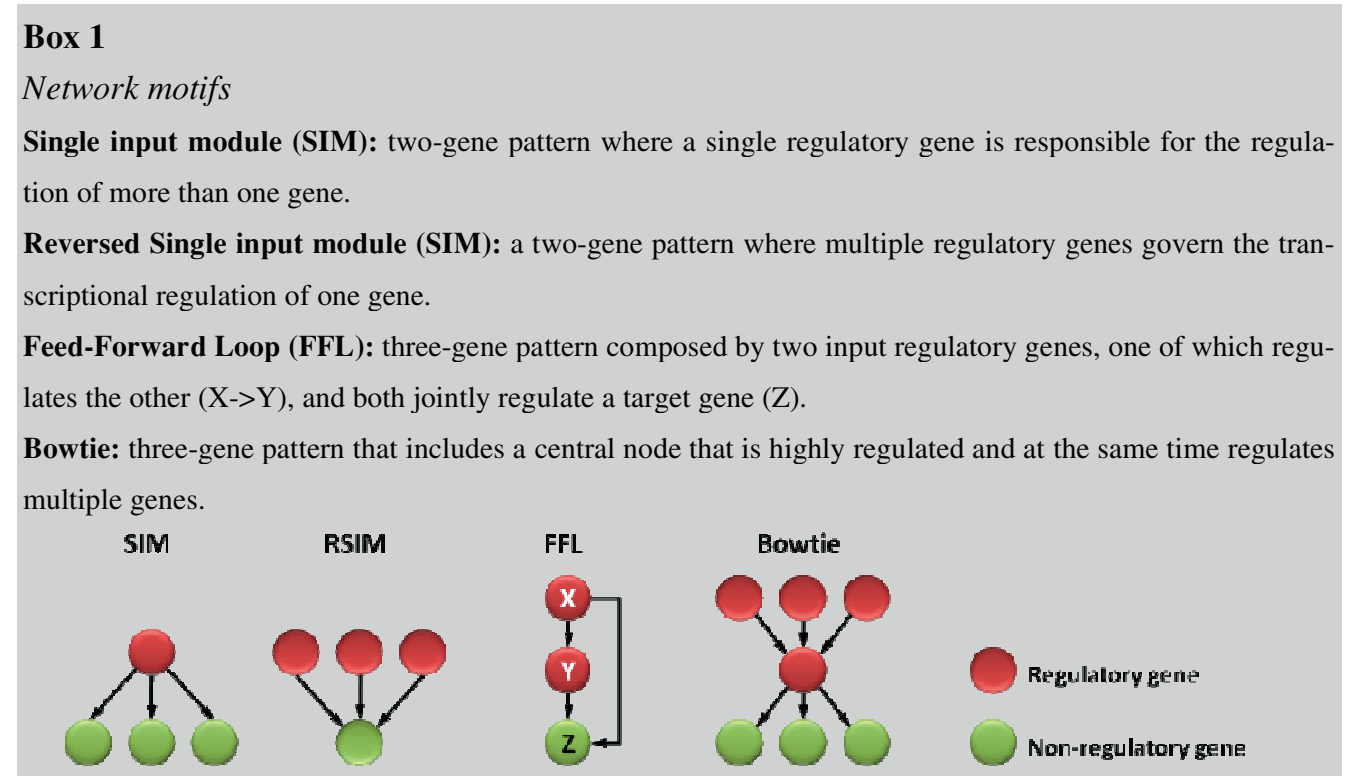

Specifically, for each pathway $\boldsymbol{P}$ and every motif type $\boldsymbol{T}$, we count the number of motifs that affect at least one gene in the pathway and denote it by $\boldsymbol{A} \boldsymbol{b} s \_\boldsymbol{F r e q} \_\boldsymbol{T} \_\boldsymbol{i n} \_\boldsymbol{P}$ (absolute frequency of motif type $\boldsymbol{T}$ in pathway $\boldsymbol{P}$ ). We then calculate the relative frequency of motif type $\boldsymbol{T}$ in $\boldsymbol{P}$, i.e. the number of times the motif type $\boldsymbol{T}$ occurs in the pathway (affects at least one of its genes) divided by the number of times it occurs in the network, as:

$$
\text { Rel_Freq_T_P }=\text { Abs_Freq_T_P / (\# of motifs of type T in the network) }
$$

Considering every gene $\boldsymbol{G}_{\boldsymbol{P}}$ belonging to the pathway $\boldsymbol{P}$, the relative frequency of $\boldsymbol{G}_{\boldsymbol{P}}$ in motif type $\boldsymbol{T}$ denotes how many genes in the pathway, in average, are expected to be included in at least one occurrence of such motif:

$$
\boldsymbol{R e l} \boldsymbol{F} \boldsymbol{r e q} \boldsymbol{G}_{\boldsymbol{P}} \boldsymbol{T} \text { : Count all_genes_in_motif_T_P/\# of genes_in_P }
$$

\section{Results and Discussion}

\subsection{Types of Regulation}

As illustrated in Fig. 1, transcriptional regulation as the only form of regulation is dominant in the E. coli metabolism (37\% of genes are regulated at least by one $\mathrm{TF}$ ), although co-regulation (i.e., the combination of transcriptional and enzymatic regulation) and enzymatic regulation are also present (16\% and 13\% of genes, respectively). This evidences that metabolic activities do not necessarily correlate proportionally with the gene expression levels of the corresponding enzymes, but are also dependent, in almost $30 \%$ of the cases, on immediate control over the enzymes.

The high percentage of unknown regulation (37\%) can be explained by the insufficiency of knowledge (it has not yet been possible to assign a function to 
approximately one third of the proteins identified in E.coli and many details are still missing from its biochemical characterisation [8]) and the non-inclusion of other regulatory mechanisms in this study (e.g. posttranslational modifications and ribosome-mediated transcriptional attenuations).

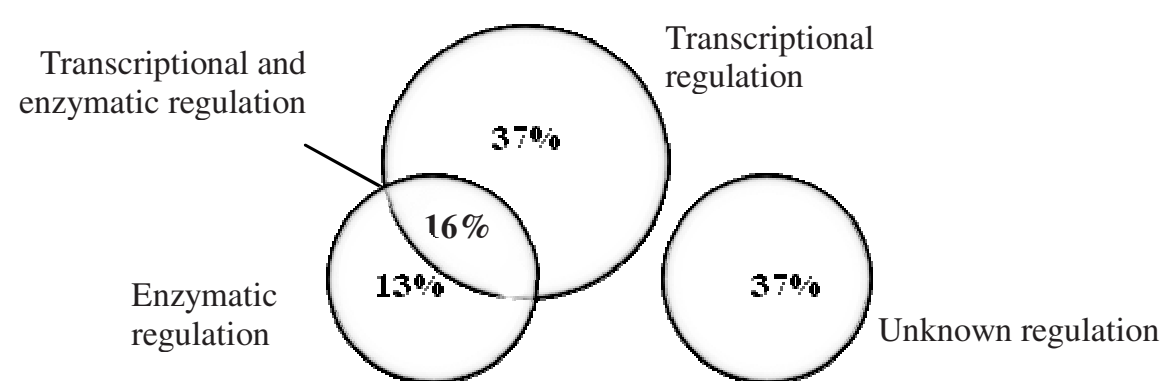

Fig. 1 The different types of regulation in the E. coli network.

Details on the regulation per pathway (Table 1) enables the generation of hypotheses about the mechanisms coordinating key metabolic processes. The transcriptional regulation is dominant in pathways like "Nitrogen Metabolism", "Citric Acid Cycle", "Inorganic Ion Transport and Metabolism" and other transport pathways. These pathways have in common the fact that their response to environmental inputs, such as availability of nitrogen and carbon sources, requires long-term regulation, i.e. modifications at gene expression level, to be able to adjust their activities accordingly. On the other hand, the existence of co-regulation in amino acids biosynthesis pathways, like "Glutamate Metabolism", "Tyrosine, Tryptophan, and Phenylalanine Metabolism" and "Arginine and Proline Metabolism", can be explained by the need to ensure both a longer-term regulation and the fine-tuning of metabolic activities coupled with the rapid response to over-accumulation of end-products.

\subsection{Regulatory Motifs}

Many metabolic pathways are dependent on the activity of transcriptional regulators that are often organized as regulatory structures or motifs acting as specific functional modules (Table 2). The association of certain structures with particular metabolic activities can be hypothesized as a consequence of specific information processing. Besides the biological relevance of these regulatory structures to modulate the activity of numerous biochemical functions, it is important to recognize that often motif overlap reveals that some genes respond to multiple regulatory mechanisms.

SIM and RSIM motifs are simple regulatory structures required to coordinate the activity of multiple genes at the metabolic level. While SIMs represent a set of genes that are controlled by a single TF (i.e. one-to-many), RSIMs display a single gene being controlled by multiple regulators (i.e. many-to-one). That is, many metabolic activities are dependent on the regulation of a single regulator (SIMs) or 
Table 1 Effect of different types of regulation in E. coli pathways. The gradient of colours illustrates the incidence of a given type of regulation in a pathway (increasing incidence ranges from black to red).

\begin{tabular}{|c|c|c|c|c|c|}
\hline Pathway & $\begin{array}{l}\text { Number of } \\
\text { Genes }\end{array}$ & $\begin{array}{l}\text { Transcriptional } \\
\text { regulation }\end{array}$ & $\begin{array}{l}\text { Metabolic } \\
\text { regulation }\end{array}$ & $\begin{array}{l}\text { Transcriptional and } \\
\text { metabolic regulation }\end{array}$ & $\begin{array}{l}\text { Unknown } \\
\text { regulation }\end{array}$ \\
\hline Alanine and Aspartate Metabolism & 11 & $27 \%$ & $\%$ & $27 \%$ & $36 \%$ \\
\hline Alternate Carbon Metabolism & 159 & $48 \%$ & $\%$ & $21 \%$ & $26 \%$ \\
\hline Anaplerotic Reactions & 10 & $20 \%$ & $40 \%$ & $20 \%$ & $20 \%$ \\
\hline Arginine and Proline Metabolism & 40 & $23 \%$ & $18 \%$ & $40 \%$ & $20 \%$ \\
\hline Cell Envelope Biosynthesis & 53 & $17 \%$ & $17 \%$ & $11 \%$ & $55 \%$ \\
\hline Citric Acid Cycle & 18 & $83 \%$ & $0 \%$ & $11 \%$ & $6 \%$ \\
\hline Cofactor and Prosthetic Group Biosynthesis & 136 & $20 \%$ & $16 \%$ & $7 \%$ & $57 \%$ \\
\hline Cysteine Metabolism & 20 & $35 \%$ & $15 \%$ & $20 \%$ & $30 \%$ \\
\hline Folate Metabolism & 4 & $25 \%$ & $50 \%$ & $25 \%$ & $0 \%$ \\
\hline Glutamate Metabolism & 11 & $0 \%$ & $9 \%$ & $64 \%$ & $27 \%$ \\
\hline Glycerophospholipid Metabolism & 20 & $15 \%$ & $35 \%$ & $0 \%$ & $50 \%$ \\
\hline Glycine and Serine Metabolism & 15 & $27 \%$ & $7 \%$ & $40 \%$ & $27 \%$ \\
\hline Glycolysis/Gluconeogenesis & 32 & $31 \%$ & $13 \%$ & $28 \%$ & $28 \%$ \\
\hline Glyoxylate Metabolism & 4 & $25 \%$ & $0 \%$ & $25 \%$ & $50 \%$ \\
\hline Histidine Metabolism & 9 & $0 \%$ & $22 \%$ & $11 \%$ & $67 \%$ \\
\hline Information Transfer & 167 & $56 \%$ & $0 \%$ & $0 \%$ & $44 \%$ \\
\hline Inorganic Ion Transport and Metabolism & 67 & $76 \%$ & $\%$ & $0 \%$ & $22 \%$ \\
\hline Lipopolysaccharide Biosynthesis / Recycling & 46 & $7 \%$ & & $2 \%$ & $83 \%$ \\
\hline Membrane Lipid Metabolism & 14 & $50 \%$ & $14 \%$ & $7 \%$ & $29 \%$ \\
\hline Methionine Metabolism & 13 & $31 \%$ & $0 \%$ & $23 \%$ & $46 \%$ \\
\hline Methylglyoxal Metabolism & 10 & $0 \%$ & $20 \%$ & $10 \%$ & $70 \%$ \\
\hline Murein Biosynthesis & 10 & $20 \%$ & & $0 \%$ & $80 \%$ \\
\hline Murein Recycling & 34 & $29 \%$ & & $\%$ & $65 \%$ \\
\hline Nitrogen Metabolism & 15 & $87 \%$ & $0 \%$ & $7 \%$ & $7 \%$ \\
\hline Nucleotide Salvage Pathway & 57 & $19 \%$ & $26 \%$ & $19 \%$ & $35 \%$ \\
\hline Oxidative Phosphorylation & 77 & $65 \%$ & $8 \%$ & $5 \%$ & $22 \%$ \\
\hline Pentose Phosphate Pathway & 13 & $15 \%$ & $31 \%$ & $23 \%$ & $31 \%$ \\
\hline Purine and Pyrimidine Biosynthesis & 22 & $41 \%$ & $18 \%$ & $27 \%$ & $14 \%$ \\
\hline Pyruvate Metabolism & 20 & $50 \%$ & $15 \%$ & $15 \%$ & $20 \%$ \\
\hline Threonine and Lysine Metabolism & 19 & $11 \%$ & $37 \%$ & $21 \%$ & $32 \%$ \\
\hline Transport, Inner Membrane & 216 & $72 \%$ & $0 \%$ & $1 \%$ & $26 \%$ \\
\hline Transport, Outer Membrane & 21 & $81 \%$ & & $0 \%$ & $19 \%$ \\
\hline Transport, Outer Membrane Porin & 4 & $75 \%$ & $0 \%$ & $0 \%$ & $25 \%$ \\
\hline tRNA Charging & 24 & $0 \%$ & $17 \%$ & $4 \%$ & $79 \%$ \\
\hline Tyrosine, Tryptophan, and Phenylalanine Metabolism & 24 & $29 \%$ & $4 \%$ & $29 \%$ & $38 \%$ \\
\hline Unassigned & 23 & $39 \%$ & $4 \%$ & $13 \%$ & $43 \%$ \\
\hline Valine, Leucine, and Isoleucine Metabolism & 17 & $76 \%$ & $0 \%$ & $18 \%$ & $6 \%$ \\
\hline
\end{tabular}

are subjected to regulation from various regulators (RSIMs). Pathways like "Alternate Carbon Metabolism" and "Transport, Inner Membrane" are examples of metabolic functions that depend on this immediate form of regulation to respond to external stimuli (e.g. nutrient carbon sources).

Bowtie structures can be interpreted as the coupling of SIM and RSIM motifs through a single central element, suggesting that these structures share a similar conceptual and architectural design. The capacity of central nodes to admit variability of input information (i.e. regulation from other genetic elements), confers high flexibility and robustness to the system, while supporting the modulation of multiple pathways simultaneously [9]. Pathways like "Transport, Inner Membrane" and "Information Transfer", which support inherently complex information exchange processes, are in need of these regulatory structures to guarantee the adequate propagation of the information throughout the network. 
Table 2 Statistical evaluation of the occurrence of regulatory motifs per pathway. Abbreviations: $T_{-} P$, relative frequency of a motif type $T$ in pathway $P ; G p_{-} T$, frequency of genes from the pathway $P(G p)$ involved in motif type $T$ (see section Network Analysis for details on the metrics used).

\begin{tabular}{|c|c|c|c|c|c|c|c|c|c|c|c|c|}
\hline \multirow{2}{*}{ Pathway } & \multicolumn{3}{|c|}{ FFL } & \multicolumn{3}{|c|}{ SIM } & \multicolumn{3}{|c|}{ RSIM } & \multicolumn{3}{|c|}{ BowTie } \\
\hline & \# & $T \_P$ & $G p \_T$ & \# & $T \_P$ & $G p \_T$ & $\#$ & $T \_P$ & $G p \_T$ & $\#$ & $T \_P$ & $G p \_T$ \\
\hline Alanine and Aspartate Metabolism & 3 & 0,00 & 0,27 & 6 & 0,05 & 1,00 & 4 & 0,01 & 0,36 & 2 & 0,10 & 0,18 \\
\hline Alternate Carbon Metabolism & 144 & 0,13 & 0,91 & 60 & 0,46 & 1,74 & 82 & 0,17 & 0,52 & 9 & 0,45 & 0,21 \\
\hline Anaplerotic Reactions & 6 & 0,01 & 0,60 & 7 & 0,05 & 1,70 & 3 & 0,01 & 0,30 & - & - & - \\
\hline Arginine and Proline Metabolism & 10 & 0,01 & 0,25 & 15 & 0,12 & 1,33 & 12 & 0,02 & 0,30 & 3 & 0,15 & 0,15 \\
\hline Cell Envelope Biosynthesis & - & - & - & 9 & 0,07 & 0,38 & 4 & 0,01 & 0,08 & 1 & 0,05 & 0,02 \\
\hline Citric Acid Cycle & 61 & 0,06 & 3,39 & 17 & 0,13 & 4,11 & 17 & 0,03 & 0,94 & 6 & 0,30 & 0,89 \\
\hline $\begin{array}{l}\text { Cofactor and Prosthetic Group } \\
\text { Biosynthesis }\end{array}$ & 45 & 0,04 & 0,33 & 27 & 0,21 & 0,63 & 23 & 0,05 & 0,17 & 5 & 0,25 & 0,10 \\
\hline Cysteine Metabolism & 6 & 0,01 & 0,30 & 6 & 0,05 & 0,85 & 4 & 0,01 & 0,20 & 1 & 0,05 & 0,05 \\
\hline Folate Metabolism & 2 & 0,00 & 0,50 & 5 & 0,04 & 1,25 & 1 & 0,00 & 0,25 & - & - & - \\
\hline Glutamate Metabolism & 41 & 0,04 & 3,73 & 13 & 0,10 & 3,18 & 6 & 0,01 & 0,55 & 5 & 0,25 & 1,18 \\
\hline Glycerophospholipid Metabolism & 7 & 0,01 & 0,35 & 8 & 0,06 & 0,45 & 2 & 0,00 & 0,10 & 1 & 0,05 & 0,05 \\
\hline Glycine and Serine Metabolism & 7 & 0,01 & 0,47 & 12 & 0,09 & 1,40 & 6 & 0,01 & 0,40 & 2 & 0,10 & 0,13 \\
\hline Glycolysis/Gluconeogenesis & 15 & 0,01 & 0,47 & 10 & 0,08 & 1,06 & 8 & 0,02 & 0,25 & 3 & 0,15 & 0,09 \\
\hline Glyoxylate Metabolism & - & - & - & 1 & 0,01 & 0,50 & - & - & - & - & - & - \\
\hline Histidine Metabolism & - & - & - & 1 & 0,01 & 0,11 & - & - & - & - & - & - \\
\hline Information Transfer & 96 & 0,09 & 0,57 & & 0,00 & & 57 & 0,11 & 0,34 & 16 & 0,80 & 0,36 \\
\hline $\begin{array}{l}\text { Inorganic Ion Transport and } \\
\text { Metabolism }\end{array}$ & 38 & 0,04 & 0,57 & 28 & 0,22 & 1,54 & 26 & 0,05 & 0,39 & 5 & 0,25 & 0,39 \\
\hline $\begin{array}{l}\text { Lipopolysaccharide Biosynthesis / } \\
\text { Recycling }\end{array}$ & - & - & - & 4 & 0,03 & 0,13 & 2 & 0,00 & 0,04 & 1 & 0,05 & 0,04 \\
\hline Membrane Lipid Metabolism & 2 & 0,00 & 0,14 & 7 & 0,05 & 1,21 & 7 & 0,01 & 0,50 & 1 & 0,05 & 0,14 \\
\hline Methionine Metabolism & 3 & 0,00 & 0,23 & 6 & 0,05 & 1,00 & 6 & 0,01 & 0,46 & 1 & 0,05 & 0,08 \\
\hline Methylglyoxal Metabolism & 1 & 0,00 & 0,10 & 2 & 0,02 & 0,20 & 1 & 0,00 & 0,10 & - & - & - \\
\hline Murein Biosynthesis & - & - & - & 1 & 0,01 & 0,10 & - & - & - & - & - & - \\
\hline Murein Recycling & 2 & 0,00 & 0,06 & 8 & 0,06 & 0,59 & 6 & 0,01 & 0,18 & - & - & - \\
\hline Nitrogen Metabolism & 49 & 0,05 & 3,27 & 15 & 0,12 & 5,53 & 11 & 0,02 & 0,73 & 4 & 0,20 & 1,53 \\
\hline Nucleotide Salvage Pathway & 16 & 0,01 & 0,28 & 18 & 0,14 & 0,82 & 12 & 0,02 & 0,21 & 2 & 0,10 & 0,14 \\
\hline Oxidative Phosphorylation & 218 & 0,20 & 2,83 & 24 & 0,18 & 3,35 & 52 & 0,10 & 0,68 & 8 & 0,40 & 0,65 \\
\hline Pentose Phosphate Pathway & 3 & 0,00 & 0,23 & 8 & 0,06 & 0,77 & 3 & 0,01 & 0,23 & 2 & 0,10 & 0,15 \\
\hline $\begin{array}{l}\text { Purine and Pyrimidine Biosynthe- } \\
\text { sis }\end{array}$ & 4 & 0,00 & 0,18 & 8 & 0,06 & 1,27 & 8 & 0,02 & 0,36 & 3 & 0,15 & 0,23 \\
\hline Pyruvate Metabolism & 42 & 0,04 & 2,10 & 17 & 0,13 & 2,50 & 13 & 0,03 & 0,65 & 3 & 0,15 & 0,50 \\
\hline Threonine and Lysine Metabolism & 5 & 0,00 & 0,26 & 11 & 0,08 & 0,63 & 4 & 0,01 & 0,21 & 3 & 0,15 & 0,16 \\
\hline Transport, Inner Membrane & 229 & 0,21 & 1,06 & 86 & 0,66 & 1,93 & 111 & 0,22 & 0,51 & 18 & 0,90 & 0,39 \\
\hline Transport, Outer Membrane & 12 & 0,01 & 0,57 & 18 & 0,14 & 1,76 & 7 & 0,01 & 0,33 & 2 & 0,10 & 0,33 \\
\hline Transport, Outer Membrane Porin & 8 & 0,01 & 2,00 & 9 & 0,07 & 3,50 & 2 & 0,00 & 0,50 & 1 & 0,05 & 0,25 \\
\hline tRNA Charging & - & - & - & 1 & 0,01 & 0,04 & - & - & - & - & - & - \\
\hline $\begin{array}{l}\text { Tyrosine, Tryptophan, and Pheny- } \\
\text { lalanine Metabolism }\end{array}$ & 2 & 0,00 & 0,08 & 7 & 0,05 & 0,79 & 4 & 0,01 & 0,17 & - & - & - \\
\hline Unassigned & 17 & 0,02 & 0,74 & 16 & 0,12 & 1,26 & 7 & 0,01 & 0,30 & 4 & 0,20 & 0,35 \\
\hline $\begin{array}{l}\text { Valine, Leucine, and Isoleucine } \\
\text { Metabolism }\end{array}$ & 15 & 0,01 & 0,88 & 10 & 0,08 & 1,76 & 7 & 0,01 & 0,41 & 2 & 0,10 & 0,18 \\
\hline
\end{tabular}


FFL motifs represent again a simple form of regulation where the activities of two TF-coding genes regulate the expression of the target gene both directly and indirectly. These motifs are likely to occur when a rapid response to an external signal is required, such as shifts in carbon sources or availability of oxygen [10]. For that reason, pathways like "Oxidative Phosphorylation", "Transport, Inner Membrane" and "Alternate Carbon Metabolism" present higher abundance of these motifs. Also, most of the genes associated with these pathways are controlled by this regulatory circuit, allowing a rapid functional switch in response to a stimulus. Next, we detail some peculiar pathways in terms of regulatory mechanisms.

\subsubsection{Folate Metabolism: A Tightly Short-Term Regulated Pathway}

The folate metabolism is central to many cellular processes in E. coli, ranging from nucleotide and amino acid biosynthesis to the production of the starting amino acid residue in protein synthesis, i.e. N-formylmethionyl-tRNA(f).

The enzyme-coding genes pur $\mathrm{U}$ and folD, core of this metabolism, are only regulated enzymatically by the amino acids glycine and methionine, and coenzyme formyltetrahydrofolate (Fig. 2a). This fact is explained by the need to balance the pools of tetrahydrofolate and one-carbon tetrahydrofolate metabolites to maintain the synthesis of glycine and methionine.

The metabolic gene $m e t \mathrm{~F}$, which is associated to the reduction of folate coenzymes, is the only one to be regulated transcriptionally, specifically by a SIM structure based on the TF-coding gene met J, which is known to repress the expression of genes involved in biosynthesis and transport of methionine.

\subsubsection{Citric Acid Cycle and Nitrogen Metabolism: Two Pathways Depending on Long Term Regulation}

Both the "Citric Acid Cycle" and "Nitrogen Metabolism" relate to respiratory catalytic pathways. The "Citric Acid Cycle" is a catabolic pathway of aerobic respiration [11]. The "Nitrogen Metabolism" comprising the periplasmic nitrate reductases (Nap) and nitrite reductases (Nrf), encoded by the napFDAGHBC and $n r f \mathrm{ABCD}$ operons respectively, supports cell growth via nitrate/nitrite respiration under anaerobic conditions $[12,13]$.

Unlike the previous example, these pathways do not have much incidence of enzymatic regulation. The isocitrate dehydrogenase is the only enzyme in the "Citric Acid Cycle" with enzymatic regulation and is subjected to allosteric control by oxaloacetate, i.e. the end product of the "Citric Acid Cycle", and glyoxylate, i.e. an anabolic intermediary that is present under specific physiological conditions (Fig. 2b). This modulation enables cells to make rapid shifts between "Citric Acid Cycle" and "Glyoxylate Metabolism" pathways and thus, adjust cellular growth to different carbon sources.

Most enzymes in both pathways are controlled via transcriptional regulation, in particular via FFL and SIM structures. For example, the suc $\mathrm{AB}$ operon in the "Citric Acid Cycle" and the napABCDGH and nrfABCD operons in "Nitrogen Metabolism" are regulated by multiple TFs through FFLs (Figs. 2b and 2c). This 
suggests that changes in the environmental conditions are counteracted by the combined action of multiple regulators, such as CRP, IHF, ArcA, FNR or Fis.

\subsubsection{Glutamate Metabolism: A Pathway Depending on Co-regulation}

The "Glutamate metabolism" was highlighted for discussion due to the significant co-occurrence of transcriptional and enzymatic regulation. This is a very important pathway, because glutamate is a major constituent of the proteins in E. coli and it is a major nitrogen donor for other biosynthesis activities.

One possible example of the complexity of this pathway is the activity of the two glutamate decarboxylases that participate in the acid resistance system controlling responses to low $\mathrm{pH}$ [14]. The coding genes gadA and gad $\mathrm{B}$ are regulated by multiple TFs, and FFLs are the common regulatory structure (Fig. 2d). The enzymes are also affected by various metabolic regulators. Intermediates of the "Citric Acid Cycle", such as fumarate and succinate, act upon these enzymes to inhibit their activity when $\mathrm{pH}$ is back normal. These combined actions enable the cell to respond quickly to $\mathrm{pH}$ perturbations.

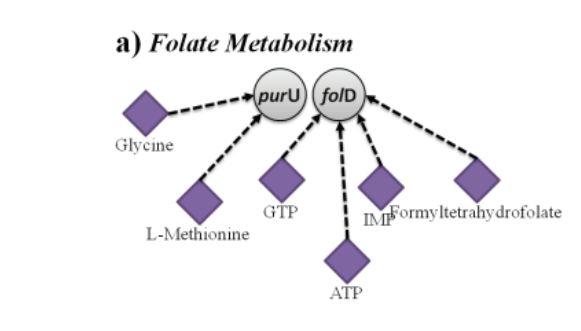

d) Glutamate Metabolism

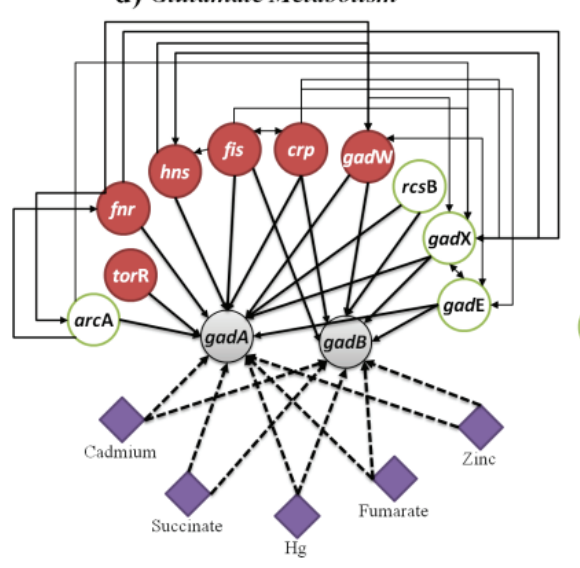

b) Citric Acid Cycle
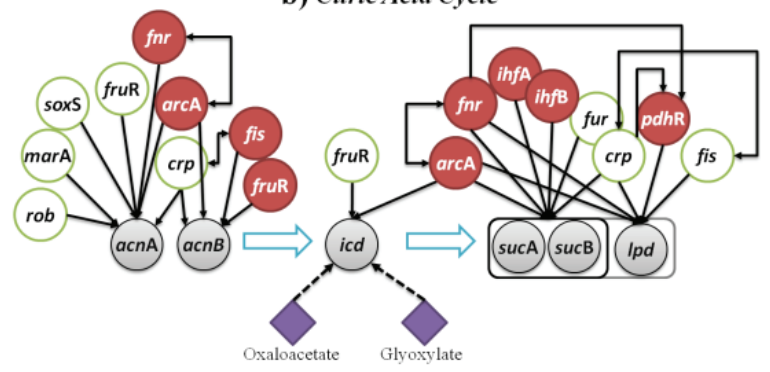

c) Nitrogen Metabolism

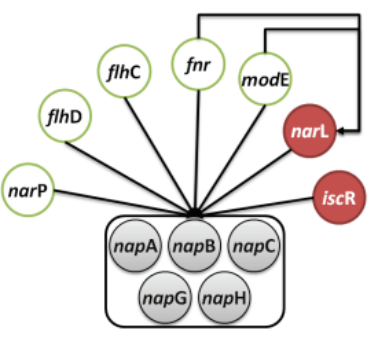

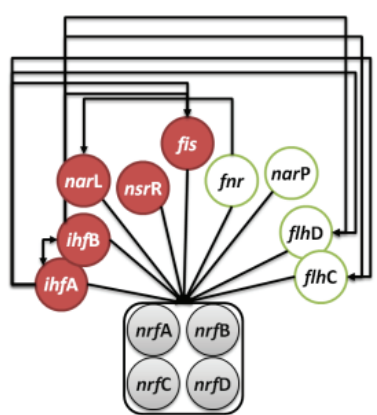

Fig. 2 Simplified representations of four pathways exhibiting particular regulatory motifs.

\section{Conclusions}

Considering that TFs and metabolic regulators have similar functional purposes, i.e. to ultimately regulate the activity of enzymatic reactions, the integrated analysis of their activities provides a new perspective over the capacity of E. coli to modulate metabolic pathways. TF-based regulation is meant to perform system 
adaption whereas enzymatic regulation is chosen when a rapid shift in a given metabolic activity is needed. For example, the shift from aerobic respiration to anaerobic respiration requires gene expression adjustments as the cells have to adapt their entire metabolism to a new environment. In turn, enzymatic regulators are needed to balance certain metabolic pools and thus maintaining the concentration of end products within acceptable ranges.

Information processing is supported by various structures of regulation, capable of responding to one or more environmental/internal inputs. Each structure has a unique way to process information (it may receive multiple inputs and/or it may affect multiple gene targets) and its relevance over a pathway is given by the number of affected genes that belong to the pathway. This view is meant to discriminate between pathways that are heavily regulated and those where TF regulation is scarce. Additionally, the study has shown that certain regulatory structures are characteristic of a subset of pathways. In particular, the ability to accept a wide range of inputs and to convey information through a single node affecting several functional elements is seen in pathways where the transference of information is critical.

Acknowledgments. This work is partly funded by the Portuguese FCT (Fundação para a Ciência e Tecnologia) funded MIT-Portugal Program in Bioengineering (MIT-Pt/BS$\mathrm{BB} / 0082 / 2008)$. The work of Sónia Carneiro and José P. Pinto are supported by $\mathrm{PhD}$ grants from FCT (ref. SFRH/BD/22863/2005 and SFRH/BD/41763/2007, respectively).

\section{References}

1. Thiele, I., Jamshidi, N., Fleming, R.M., Palsson, B.O.: Genome-scale reconstruction of Escherichia coli's transcriptional and translational machinery: a knowledge base, its mathematical formulation, and its functional characterization. PLoS Comput. Biol. 5, e1000312 (2009)

2. Abdul Kadir, T.A., Mannan, A.A., Kierzek, A.M., McFadden, J., Shimizu, K.: Modeling and simulation of the main metabolism in Escherichia coli and its several singlegene knockout mutants with experimental verification. Microb. Cell Fact. 9, 88 (2010)

3. Feist, A.M., Palsson, B.O.: The growing scope of applications of genome-scale metabolic reconstructions using Escherichia coli. Nat. Biotechnol. 26, 659-667 (2008)

4. Feist, A.M., Henry, C.S., Reed, J.L., Krummenacker, M., Joyce, A.R., Karp, P.D., Broadbelt, L.J., Hatzimanikatis, V., Palsson, B.O.: A genome-scale metabolic reconstruction for Escherichia coli K-12 MG1655 that accounts for 1260 ORFs and thermodynamic information. Mol. Syst. Biol. 3, 121 (2007)

5. Keseler, I.M., Collado-Vides, J., Santos-Zavaleta, A., Peralta-Gil, M., Gama-Castro, S., Muniz-Rascado, L., Bonavides-Martinez, C., Paley, S., Krummenacker, M., Altman, T., Kaipa, P., Spaulding, A., Pacheco, J., Latendresse, M., Fulcher, C., Sarker, M., Shearer, A.G., Mackie, A., Paulsen, I., Gunsalus, R.P., Karp, P.D.: EcoCyc: a comprehensive database of Escherichia coli biology. Nucleic Acids Res. (2010)

6. Shen-Orr, S.S., Milo, R., Mangan, S., Alon, U.: Network motifs in the transcriptional regulation network of Escherichia coli. Nat. Genet. 31, 64-68 (2002)

7. Alon, U.: Network motifs: theory and experimental approaches. Nat. Rev. Genet. 8, 450-461 (2007) 
8. Hu, P., Janga, S.C., Babu, M., Diaz-Mejia, J.J., Butland, G., Yang, W., Pogoutse, O., Guo, X., Phanse, S., Wong, P., Chandran, S., Christopoulos, C., Nazarians-Armavil, A., Nasseri, N.K., Musso, G., Ali, M., Nazemof, N., Eroukova, V., Golshani, A., Paccanaro, A., Greenblatt, J.F., Moreno-Hagelsieb, G., Emili, A.: Global functional atlas of Escherichia coli encompassing previously uncharacterized proteins. PLoS Biol. 7, e96 (2009)

9. Ma, H.W., Zhao, X.M., Yuan, Y.J., Zeng, A.P.: Decomposition of metabolic network into functional modules based on the global connectivity structure of reaction graph. Bioinformatics 20, 1870-1876 (2004)

10. Shen-Orr, S.S., Milo, R., Mangan, S., Alon, U.: Network motifs in the transcriptional regulation network of Escherichia coli. Nat. Genet. 31, 64-68 (2002)

11. Shalel-Levanon, S., San, K.Y., Bennett, G.N.: Effect of oxygen, and ArcA and FNR regulators on the expression of genes related to the electron transfer chain and the TCA cycle in Escherichia coli. Metab. Eng. 7, 364-374 (2005)

12. Brondijk, T.H., Nilavongse, A., Filenko, N., Richardson, D.J., Cole, J.A.: NapGH components of the periplasmic nitrate reductase of Escherichia coli K-12: location, topology and physiological roles in quinol oxidation and redox balancing. Biochem. J. 379, 47-55 (2004)

13. van Wonderen, J.H., Burlat, B., Richardson, D.J., Cheesman, M.R., Butt, J.N.: The nitric oxide reductase activity of cytochrome c nitrite reductase from Escherichia coli. J. Biol. Chem. 283, 9587-9594 (2008)

14. Castanie-Cornet, M.P., Cam, K., Bastiat, B., Cros, A., Bordes, P., Gutierrez, C.: Acid stress response in Escherichia coli: mechanism of regulation of gadA transcription by RcsB and GadE. Nucleic Acids Res. 38, 3546-3554 (2010) 